Acta Crystallographica Section F

Structural Biology

and Crystallization

Communications

ISSN 1744-3091

\author{
Stephen N. Hewitt, ${ }^{a, b}$ Ryan \\ Choi, ${ }^{a, b}$ Angela Kelley, ${ }^{a, b}$ \\ Gregory J. Crowther, ${ }^{b}$ Alberto J. \\ Napuli ${ }^{a, b}$ and Wesley C. Van \\ Voorhis $^{\mathrm{a}, \mathrm{b}_{*}}$
}

${ }^{\text {a }}$ Seattle Structural Genomics Center for Infectious Disease (SSGCID), University of Washington, WA 98195, USA, and ${ }^{\mathbf{b}}$ Division of

Allergy and Infectious Diseases, School of Medicine, Box 356423, University of

Washington, Seattle, WA 98195-6423, USA

Correspondence e-mail: wesley@uw.edu

Received 11 February 2011

Accepted 7 June 2011

\section{Expression of proteins in Escherichia coli as fusions with maltose-binding protein to rescue non-expressed targets in a high-throughput protein-expression and purification pipeline}

Despite recent advances, the expression of heterologous proteins in Escherichia coli for crystallization remains a nontrivial challenge. The present study investigates the efficacy of maltose-binding protein (MBP) fusion as a general strategy for rescuing the expression of target proteins. From a group of sequence-verified clones with undetectable levels of protein expression in an E. coli $\mathrm{T} 7$ expression system, 95 clones representing 16 phylogenetically diverse organisms were selected for recloning into a chimeric expression vector with an N-terminal histidine-tagged MBP. PCR-amplified inserts were annealed into an identical ligation-independent cloning region in an MBP-fusion vector and were analyzed for expression and solubility by high-throughput nickel-affinity binding. This approach yielded detectable expression of $72 \%$ of the clones; soluble expression was visible in $62 \%$. However, the solubility of most proteins was marginal to poor upon cleavage of the MBP tag. This study offers large-scale evidence that MBP can improve the soluble expression of previously nonexpressing proteins from a variety of eukaryotic and prokaryotic organisms. While the behavior of the cleaved proteins was disappointing, further refinements in MBP tagging may permit the more widespread use of MBPfusion proteins in crystallographic studies.

\section{Introduction}

The process of solving three-dimensional protein structures by X-ray crystallography is a multi-stage effort with unique challenges at each step. The initial challenge is expression of the protein in a sufficient quantity and with sufficient purity to obtain diffraction-quality crystals. Typically, recombinant proteins are expressed in Escherichia coli with small affinity tags such as hexahistidine (His tag) to allow efficient separation of the desired protein by affinity chromatography (Arnold, 1991). E. coli expression systems are a proven and costeffective method of producing large quantities of high-quality recombinant proteins (Arnold, 1991; Gathmann et al., 2006; MusVeteau, 2002). However, this approach is often insufficient for soluble expression of recombinant protein. The Structural Genomics Center has estimated that up to $50 \%$ of all prokaryotic proteins are insoluble when expressed in E. coli (Edwards et al., 2000). Failure to express soluble eukaryotic proteins is even more common; for instance, reports indicate that only 6-20\% of human and Plasmodium proteins are solubly expressed using standard expression methods (Stevens, 2000; Mehlin et al., 2006). Insoluble protein expression is thus a major impediment to structural genomics efforts.

One strategy for increasing expression and solubility is fusing the target protein to a large affinity tag such as glutathione $S$-transferase (GST), thioredoxin (TRX) or maltose-binding protein (MBP) (Smith, 2000; Sachdev \& Chirgwin, 2000; LaVallie et al., 2000; Kapust \& Waugh, 1999). Of these, the most promising may be MBP. Although unaltered MBP can be purified using a cross-linked amylose resin affinity matrix, addition of a His tag or GST improves the yield and purity (Pryor \& Leiting, 1997). His-tagged MBP-fusion constructs therefore allow metal-affinity purification strategies combined with the potential for increased solubility (di Guan et al., 1988).

Previous studies have provided preliminary evidence of the ability of MBP to rescue the expression and solubility of proteins. In a comparison of three macromolecule chimeric constructs, MBP was 


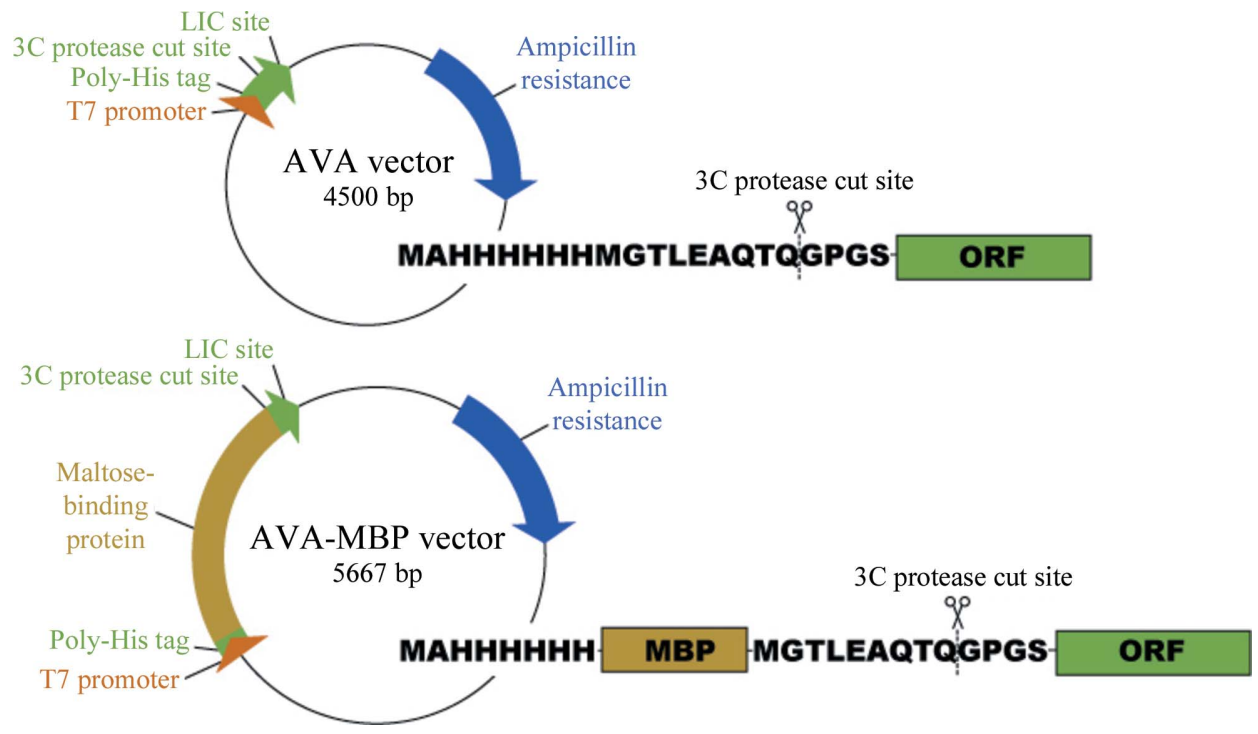

Figure 1

Comparison of AVA vector and AVA-MBP vector.

far more effective than GST or TRX in solubilizing six notoriously insoluble proteins (Kapust \& Waugh, 1999). In a study of 32 small human proteins expressed in E. coli in tandem with MBP, researchers observed increased solubility and expression in 19 out of 32 constructs compared with His-tag expression (Hammarström et al., 2002). In an examination of membrane proteins from Mycobacterium tuberculosis, the expression of 16 out of 22 proteins was rescued by fusion to MBP and 13 of these 16 were soluble (Korepanova et al., 2007). A larger study by Kataeva et al. (2005) observed increased soluble expression levels in 60 out of 66 Clostridium thermocellum and 38 out of 79 Shewanella oneidensis proteins when targets were expressed fused to MBP in combination with decreased induction temperatures, compared with the expression of GST or NusA fusion partners. To our knowledge, however, a large-scale study of the effects of MBP on proteins from diverse eukaryotic and prokaryotic organisms has yet to be reported.

The goal of this study was to examine fusion proteins on a scale permitting a highly accurate assessment of the rescue rate of MBP. We attempted MBP-mediated rescue of 95 His-tagged targets from diverse sources. These targets had no soluble or total expression but were sequence-validated, ensuring that the lack of expression was not attributable to an incorrect target or an empty vector.

\section{Methods}

Targets for the SSGCID pipeline were selected for their predicted essentiality, virulence factor and general potential as drug targets. For cloning, targets were amplified from either purified genomic DNA or cDNA using standardized primers containing sequences specific for the cloning site followed by a sequence complementary (adjusted to $331 \mathrm{~K} T_{\mathrm{m}}$ ) to the template gene: FWD primer $5^{\prime}$-GGGTCCTGGTTCATG ... - - $3^{\prime}$ and REV primer 5'-CTTGTTCGTGCTGTTTATTA ... - - $3^{\prime}$ (Invitrogen). PCR conditions were optimized depending on the $\mathrm{G}+\mathrm{C}$ content of the template sequence. A typical reaction

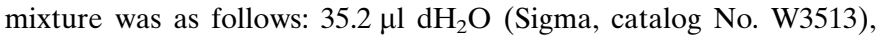
$5 \mu$ l Expand High Fidelity Buffer $(10 \times)$ with $15 \mathrm{mM} \mathrm{MgCl}$ (Roche), $0.4 \mu \mathrm{l} 25 \mathrm{~m} M$ dNTPs (Qiagen, catalog No. 201912), $0.4 \mu$ l Expand High Fidelity Enzyme Mix (Roche, catalog No. 11732641001), $4 \mu \mathrm{l}$ $(10 \mu M)$ FWD primer, $4 \mu \mathrm{l}(10 \mu M)$ REV primer and $1 \mu \mathrm{l}(10 \mathrm{ng})$ of the respective template DNA. PCR amplicons were run on a $1 \%$ agarose gel to verify the expected size of the amplified gene; the band was then excised from the gel and extracted from the agarose using a QiaQuick kit (Qiagen, catalog No. 28181). The purified PCR product was cloned into expression vector AVA0421 (which expresses protein with a hexahistidine-tag fusion that is cleavable with $3 \mathrm{C}$ protease to leave a minimal four-amino-acid sequence at the $\mathrm{N}$-terminus) or AVA-MBP (which expresses protein with an $\mathrm{N}$-terminal hexahistidine tag in tandem with MBP that is cleavable with $3 \mathrm{C}$ protease) by ligation-independent cloning (LIC; Aslanidis \& De Jong, 1990; Fig. 1). Briefly, purified PCR product was treated with T4 polymerase in the presence of the single nucleotide dATP, creating overhangs, and then annealed with compatible, linearized and T4-treated AVA0421 vector (Mehlin et al., 2006). Annealed vector and insert were transformed into NovaBlue competent cells (Novagen, catalog No. 71011-4) and plated on LB agar (BD Difco LB Agar Miller; BD, catalog No. 244520 ) with $50 \mu \mathrm{g} \mathrm{ml}^{-1}$ each of ampicillin (Anatrace, catalog No. A1000) and carbenicillin (Duchefa Biochemie, catalog No. C0109.0025) to select for cells carrying the expression plasmid. The presence of the insert was verified by colony PCR (using the above conditions but the colony was resuspended in water and used as template instead of purified DNA). Plasmid DNA was purified (QIAprep Turbo mini-prep kit; Qiagen, catalog No. 27191) from $1 \mathrm{ml}$

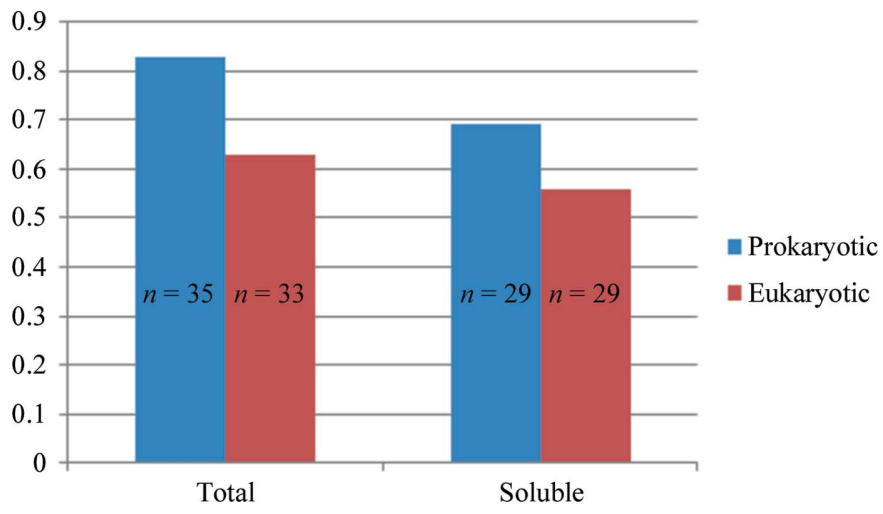

Figure 2

Distribution of protein solubility by species, grouped by prokaryotic and eukaryotic kingdoms. 


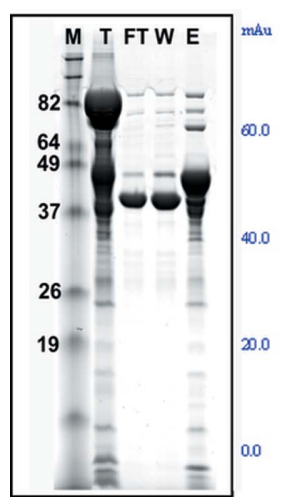

(a)

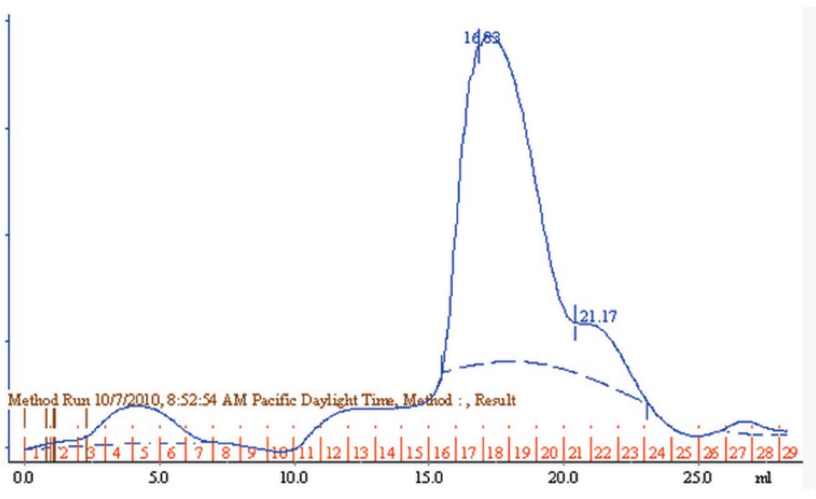

(b)

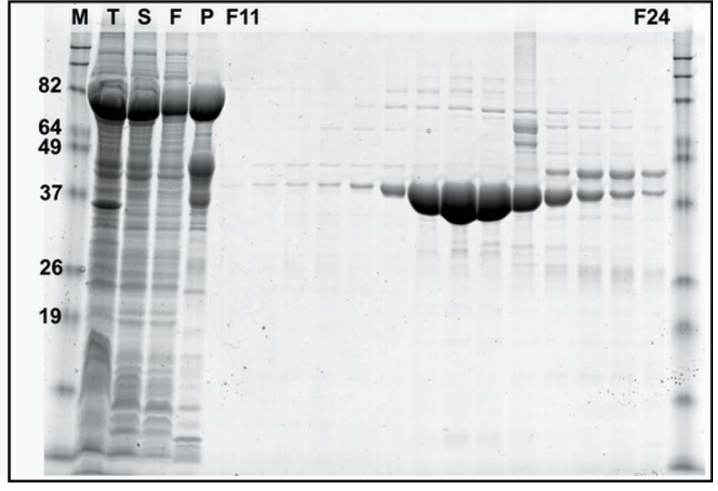

(c)

Figure 3

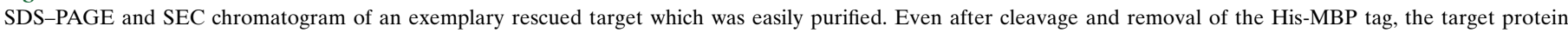

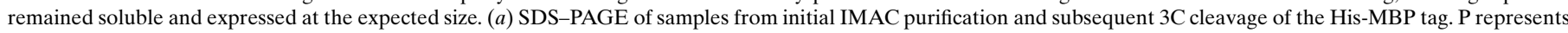

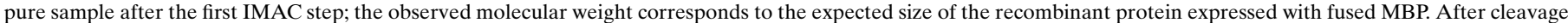

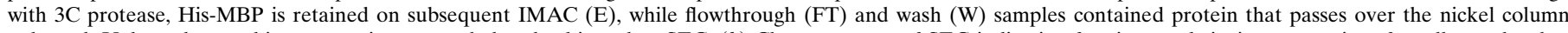

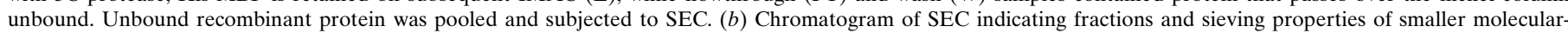

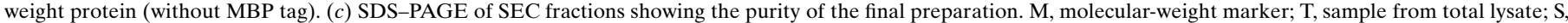
sample from soluble fraction after centrifugation. The protein expressed and purified was an uncharacterized protein from Coccidioides immitis.

overnight cultures and then transformed into the expression host Rosetta Oxford [BL21 Star(DE3)-R3-pRARE2] (Choi et al., 2011).

For protein purification, 21 cultures of the clone were grown in a LEX bioreactor (Harbinger) at $293 \mathrm{~K}$ in auto-induction medium (Studier, 2005). After $72 \mathrm{~h}$ of growth, the culture was pelleted (Sorvall RC 12BP fitted with an H-12000 rotor; spun for $20 \mathrm{~min}$ at $4300 \mathrm{rev}^{-1}$ ) and the cell paste was harvested and flash-frozen in liquid nitrogen. To prepare protein samples, the cell paste was solubilized in $200 \mathrm{ml}$ lysis buffer (25 $\mathrm{m} M$ HEPES, $500 \mathrm{~m} M \mathrm{NaCl}, 5 \%$ glycerol, $30 \mathrm{~m} M$ imidazole, $0.5 \%$ CHAPS, $10 \mathrm{~m} M \mathrm{MgCl}_{2}, 1 \mathrm{~m} M$ TCEP, $25 \mu \mathrm{g} \mathrm{ml}^{-1}$ AEBSF pH 7.0) with $0.01 \mathrm{~g}$ lysozyme and sonicated for $30 \mathrm{~min}(100 \mathrm{~W}$, cycles of $15 \mathrm{~s}$ pulse-on and $15 \mathrm{~s}$ pulse-off; Virtis, catalog No. 408912). After sonication, the samples were treated with benzonase $(500 \mathrm{U})$ and then centrifuged for $1 \mathrm{~h}$ (14 $000 \mathrm{rev} \mathrm{min}^{-1}$ in a Sorvall SLA-1500 rotor) to clarify the cell debris. The protein was purified by immobilized metal ion-affinity chromatography on pre-equilibrated (25 $\mathrm{m} M$ HEPES $\mathrm{pH} 7.0$, $500 \mathrm{~m} M \mathrm{NaCl}, 5 \%$ glycerol, $30 \mathrm{~m} M$ imidazole, $1 \mathrm{~m} M$ TCEP and $0.025 \%$ azide) $5 \mathrm{ml} \mathrm{Ni}$ Sepharose columns (HisTrap FF; GE Healthcare, catalog No. 17-5255-01) using an ÄTKAexplorer. After thorough washing, the bound protein was eluted from the nickel column by addition of elution buffer ( $25 \mathrm{~m} M$ HEPES pH 7.0, $500 \mathrm{~m} M$ $\mathrm{NaCl}, 5 \%$ glycerol, $1 \mathrm{~m} M$ TCEP, $250 \mathrm{~m} M$ imidazole and $0.025 \%$ azide). Fractions from nickel-affinity chromatography were analyzed for protein content and pooled. The N-terminal $6 \times$ His tag was removed by treatment with His-MBP-3C protease overnight at $277 \mathrm{~K}$ in $3 \mathrm{C}$ buffer ( $25 \mathrm{~m} M$ HEPES pH 7.0, $200 \mathrm{~m} M \mathrm{NaCl}, 5 \%$ glycerol, $1 \mathrm{~m} M$ TCEP, $0.025 \%$ azide). Cleaved protein samples were passed over Ni resin beads (Ni Sepharose 6 Fast Flow; GE Healthcare, catalog No. 17-5318-02) to remove noncleaved protein, the cleaved $6 \times$ His tag, $3 \mathrm{C}$ protease and contaminants that bind to nickel. Clarified cleaved protein was then further purified by size-exclusion chromatography (SEC; HiLoad 26/60 Superdex 75; GE Healthcare, catalog No. 17-1071-01) using an ÄTKAprime to collect fractions in SEC buffer (25 $\mathrm{m} M$ HEPES pH 7.0, $500 \mathrm{~m} M \mathrm{NaCl}, 5 \%$ glycerol, $2 \mathrm{~m} M$ DTT, $0.025 \%$ azide). SEC fractions were analyzed by SDSPAGE. The highest intensity SEC fractions were pooled and concentrated (Amicon Ultra-15 concentrator with a molecularweight cutoff of 3000 Da; Fisher, catalog No. UFC901096). Protein samples were aliquoted in $100 \mu \mathrm{l}$ volumes, flash-frozen in liquid nitrogen and stored at $193 \mathrm{~K}$.

\section{Results and discussion}

As part of the SSGCID structural genomics pipeline, we routinely analyze by SDS-PAGE both the total and soluble fractions of smallscale cultures in 96-well sets (see Choi et al., 2011) in order to identify tractable targets for purification. These high-throughput screens are analyzed to identify insoluble or non-expressing constructs and, in most cases, to remove them from the pipeline. However, the present study further pursued these cases of suboptimal expression in order to directly compare His-tag-fusion expression with MBP-fusion expression. Our specific goal was to quantity the frequency with which adding MBP to a protein increased expression and solubility sufficiently to allow purification. We modified our His-tag E. coli expression vector to include an MBP tag between the hexahistidine residues and the $3 \mathrm{C}$ cleavage recognition site (allowing cleavage of the $\mathrm{N}$-terminal tag during purification) four amino acids upstream of the methionine start signal of the target protein, while maintaining the same insertion sequence (Fig. 1). This design strategy allowed us to efficiently employ the same PCR-amplified T4 polymerase-treated product for LIC insertion into either vector without requiring further modification.

From the entire set of constructs screened for the SSGCID project in our standard expression vector with the minimal His tag, we identified 497 unique clones that had neither total nor soluble protein expression. From this group of nearly 500 targets, we verified by sequencing that 295 of the constructs contained the expected gene sequence, eliminating the possibility of an empty vector as the reason for the lack of protein expression and verifying correct PCR amplification. 95 of these genes were selected for further study based on our desire to cover a wide range of protein functions from both prokaryotic and eukaryotic organisms (see Supplementary Material ${ }^{\mathbf{1}}$ for a complete list of proteins).

\footnotetext{
${ }^{1}$ Supplementary material has been deposited in the IUCr electronic archive (Reference: EN5465).
} 


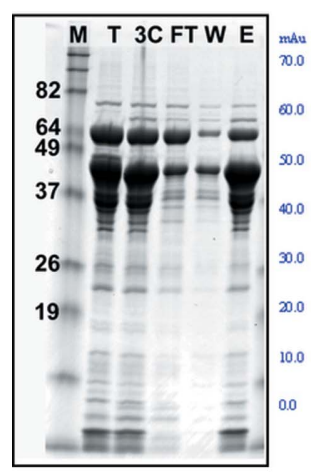

(a)

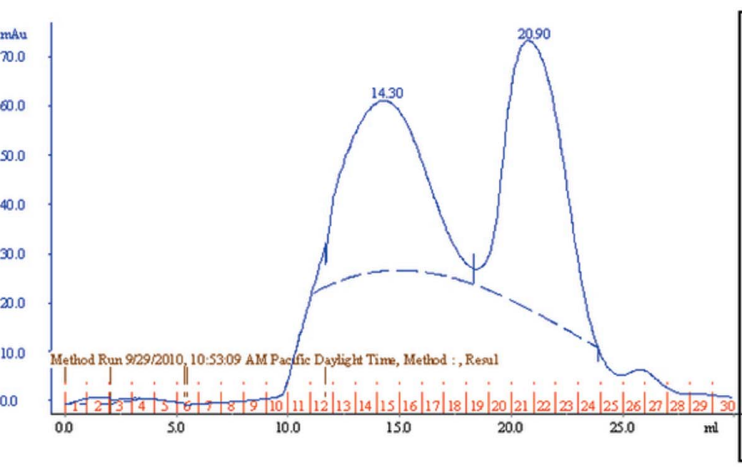

(b)

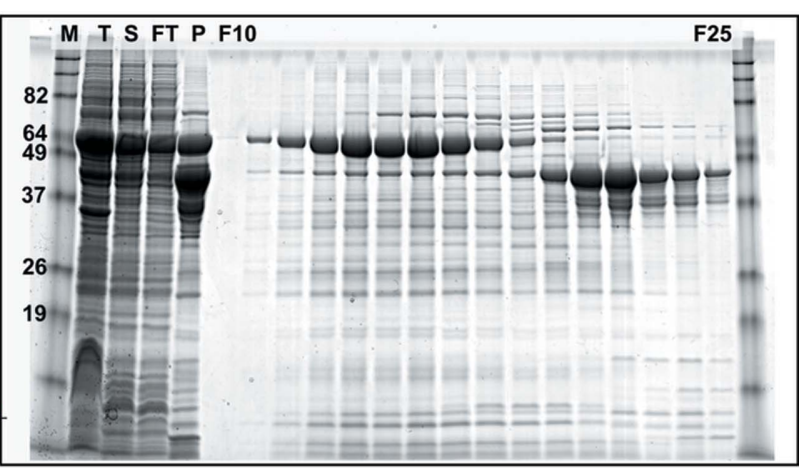

(c)

Figure 4

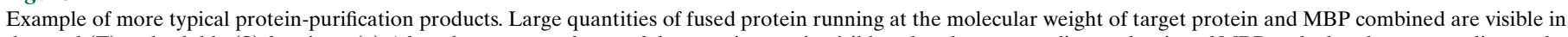

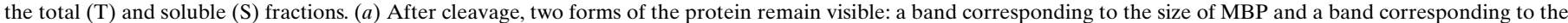

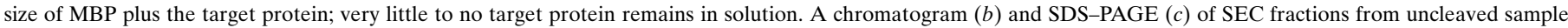

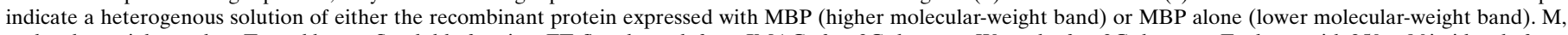

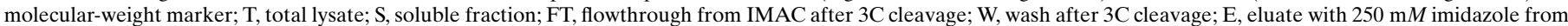
IMAC after 3C cleavage. The protein expressed and purified was Brucella abortus blue (type 1) copper protein.

Of the 95 targets selected for this study, 94 were successfully cloned into the expression host and screened for solubility. The vast majority of the targets $(72 \%)$ expressed some level of protein of the expected size (the combined size of the target protein and MBP) as visualized by SDS-PAGE. 58 targets $(62 \%)$ additionally had protein of the expected size in the soluble fraction. The majority (37) of these had detectable but low soluble expression (band easily visible on the gel), 11 samples had medium solubility (representing roughly $20-40 \%$ of soluble protein) and ten had high soluble expression levels (representing $\geq 40 \%$ of the soluble protein). Of the 36 remaining targets, 23 expressed a protein band which corresponded to the expected size of MBP alone, indicating that in these samples MBP was solubly expressed but not the fusion partner. Rescue rates were fairly similar among targets from prokaryotic and eukaryotic sources (Fig. 2).

To date, purifications have been attempted on 21 of the 58 solubly expressing proteins. 15 of these purifications yielded $>1 \mathrm{mg}$ purified protein; in 12 cases the abundance and purity of the target sample were considered to be adequate for crystallization trials (Fig. 3) and in one case crystals suitable for X-ray diffraction were obtained. This low success rate (obtaining crystals from only one of 21 attempted purifications) reflects the fact that the target proteins were usually of poor solubility after the MBP tag had been removed. Even in the 12 best purifications mentioned above yields of the MBP-free target protein were low (often 1-10 mg) and they were sometimes contaminated with significant amounts of still uncleaved protein (Fig. 4).

Insolubility may be an intrinsic property of a particular protein or may be a consequence of inadequate folding properties of the expression host. Our observations are consistent with MBP being a transiently stabilizing protein, with partner proteins falling out of solution once MBP is removed. One model is that highly soluble MBP acts as a chaperone by sequestering the aggregation-prone passenger protein, allowing native conformational folding of the nascent protein but in a weak reversible manner (Kapust \& Waugh, 1999). Recent studies have made available new fusion constructs with mutations to MBP to decrease the surface entropy and increase the rigidity of the polypeptide sequence linking MBP to the recombinant protein (Moon et al., 2010). These modifications have allowed direct structure solution by X-ray crystallography and molecular replacement without necessitating the removal of the MBP tag (Smyth et al., 2003; Moon et al., 2010). Although further advancements need to be made for this to be viable as a high-throughput rescue strategy, this approach shows promise for those recombinant protein products that could not be separated from the MBP tag by 3C cleavage. Other solubility-enhancing tags, such as SUMO expression systems, are a potential alternative for rescue of insoluble or non-expressing recombinant constructs (Yunus \& Lima, 2009).

The authors wish to thank the other members of SSGCID for their support, Christopher Mehlin and Wim Hol for initiating many of the methods described here and the other members of UW-PPG, especially Lynn K. Barrett, Janhavi Bhandari, Cassie Bryan and David Leibly. This research was funded under Federal Contract No. HHSN272200700057C from the National Institute of Allergy and Infectious Diseases, National Institutes of Health, Department of Health and Human Services.

\section{References}

Arnold, F. H. (1991). Biotechnology, 9, 151-156.

Aslanidis, C. \& de Jong, P. J. (1990). Nucleic Acids Res. 18, 6069-6074.

Choi, R., Kelley, A., Leibly, D., Nakazawa Hewitt, S., Napuli, A. \& Van Voorhis, W. (2011). Acta Cryst. F67, 998-1005.

di Guan, C., Li, P., Riggs, P. D. \& Inouye, H. (1988). Gene, 67, 21-30.

Edwards, A. M., Arrowsmith, C. H., Chrstendat, D., Dharamasi, A., Friesen, J. D., Greenblatt, J. F. \& Vedadi, M. (2000). Nature Struct. Biol. 7, 970-972. Gathmann, S., Rupprecht, E. \& Schneider, D. (2006). J. Biochem. Mol. Biol. 39, 717-721.

Hammarström, M., Hellgren, N., van den Berg, S., Berglund, H. \& Härd, T. (2002). Protein Sci. 11, 313-321.

Kapust, R. B. \& Waugh, D. S. (1999). Protein Sci. 8, 1668-1674.

Kataeva, I., Chang, J., Xu, H., Luan, C.-H., Zhou, J., Uversky, V. N., Lin, D., Horanyi, P., Liu, Z.-J., Ljungdahl, L. G., Rose, J., Luo, M. \& Wang, B.-C. (2005). J. Proteome Res. 4, 1942-1951.

Korepanova, A., Moore, J. D., Nguyen, H. B., Hua, Y., Cross, T. A. \& Gao, F. (2007). Protein Expr. Purif. 53, 24-30.

LaVallie, E. R., Lu, Z., Diblasio-Smith, E. A., Collins-Racie, L. A. \& McCoy, J. M. (2000). Methods Enzymol. 326, 322-340.

Mehlin, C. et al. (2006). Mol. Biochem. Parasitol. 148, 144-160.

Moon, A. F., Mueller, G. A., Zhong, X. \& Pedersen, L. C. (2010). Protein Sci. 19, 901-913.

Mus-Veteau, I. (2002). Comp. Funct. Genomics, 3, 511-517.

Pryor, K. D. \& Leiting, B. (1997). Protein Expr. Purif. 10, 309-319.

Sachdev, D. \& Chirgwin, J. M. (2000). Methods Enzymol. 12, 122-132.

Smith, D. B. (2000). Methods Enzymol. 326, 254-270.

Smyth, D. R., Mrozkiewicz, M. K., McGrath, W. J., Listwan, P. \& Kobe, B. (2003). Protein Sci. 12, 1313-1322.

Stevens, R. C. (2000). Struct. Fold. Des. 8, R177-R185.

Studier, F. W. (2005). Protein Expr. Purif. 41, 207-234.

Yunus, A. A. \& Lima, C. D. (2009). Methods Mol. Biol. 497, 167-186. 